University of Nebraska - Lincoln

DigitalCommons@University of Nebraska - Lincoln

Robert Katz Publications

Research Papers in Physics and Astronomy

October 1984

\title{
Some Comments on the Concepts of Dose and Dose Equivalent
}

\author{
Robert Katz \\ University of Nebraska-Lincoln, rkatz2@unl.edu \\ Werner Hofmann \\ University of Salzburg, A-5020 Salzburg, Austria
}

Follow this and additional works at: https://digitalcommons.unl.edu/physicskatz

Part of the Physics Commons

Katz, Robert and Hofmann, Werner, "Some Comments on the Concepts of Dose and Dose Equivalent" (1984). Robert Katz Publications. 109.

https://digitalcommons.unl.edu/physicskatz/109

This Article is brought to you for free and open access by the Research Papers in Physics and Astronomy at DigitalCommons@University of Nebraska - Lincoln. It has been accepted for inclusion in Robert Katz Publications by an authorized administrator of DigitalCommons@University of Nebraska - Lincoln. 
Published in Health Physics 47:4 (October 1984), pp. 603-611.

Copyright (C) 1984 Health Physics Society; published by Pergamon Press Ltd. Used by permission.

Supported by the U.S. Department of Energy

Submitted September 29, 1983; accepted June 20, 1984.

\title{
Some Comments on the Concepts of Dose and Dose Equivalent
}

\author{
Robert Katz \\ University of Nebraska-Lincoln, Lincoln, NE 68588 \\ and \\ Werner Hofmann \\ University of Salzburg, A-5020 Salzburg, Austria
}

\begin{abstract}
Although dose is the simplest and most widely used measurement of a radiation field, it does not always lead to an unambiguous estimate of response. This is reflected in the very wide range of relative biologic effectiveness (RBE) values for biological systems. The ambiguity arises from the focus on energy deposition as the source of biological effect, whether in macroscopic or microscopic volumes. The properties of the biological detector play a role equally important to the properties of the radiation field in their interaction. To predict even the most experimentally accessible biological response, cell killing, we must know the probability per unit path length for generating the observed end point. Especially for high LET radiations we need the action cross sections and the particle-energy spectrum. No one parameter reduction of a radiation field can predict biological effect. For cell killing, however, such a prediction can be made from a two-parameter reduction of the interaction between the radiation field and a specific cell line and a specific ambience of the survival curve for the specific radiation field. The determination of these two parameters leads to a suggested new procedure for evaluating the dose equivalent.
\end{abstract}

\section{Introduction}

What must we know about a radiation field to predict an observable end point, especially at low doses of ionizing radiations where the probability of observing an effect is small?

It is customary to refer all effects to measurements of dose, of the deposited energy per unit mass. One must ask these questions: "Why dose?" "Why deposited energy?" Deposited energy is certainly not the fundamental quantity, for it is always qualified as the energy of ionizing radiations. For low LET radiations, it is because the dose is related to a more fundamental quantity, the fluence of electrons in the electron slowing down spectrum.

Unfortunately most phenomena are not single-valued functions of dose. Depending on biological pa- rameters, experimental conditions, the end point, the radiation quality, the dose level, the rate, etc., the response may vary over four orders of magnitude. In radiobiology, the RBE's range from about $10^{-2}$ to $10^{2}-$ the lower values observed for sensitive 1-hit systems with very high LET radiations, and the higher values observed for cataract formation at low doses of low-energy neutrons. In physics we are accustomed to zero order relationships whose first order correction is, say, $10 \%$ or so. In radiobiology, if the zero order measurement is the response of a system to $r$ rays, the response per rad to other radiations may vary not by $10 \%$ but by factors as large as $10^{2}$. It is evident that dose, or deposited energy, is not an ideal reference parameter. Its utility lies in the fact that it is more readily measurable than other, more significant, descriptions of a radiation field. 
Despite these problems, radiation risk is usually expressed as a single-valued function of radiation dose, multiplied by the quality factor for a given radiation. In radiation protection, the prevailing area of interest is the low-dose region. For high-LET radiations, low-dose effects result from interactions of isolated particle tracks with biological matter. We therefore seek to base radiation risk on cellular radiation effects assuming that a single cell is the sensitive target for a defined biological end point, disregarding intercellular effects. Initial radiation damage at the organ level is assumed to be a mere accumulation of effects arising in single cells. Experimental evidence tells us that the probability of the final radiation effect depends on a number of modifying factors, such as repair, repopulation, cell cycle stage, etc. It has, however, generally been found that the role of these biological factors is reduced for high-LET radiations, at least for cellular inactivation. More complex disease mechanisms, such as neoplasia, require a complete mechanistic understanding which is still too fragmentary. Thus we limit our analysis to cellular inactivation where the time factor - which may play an important role in carcinogenesis - is of less significance.

For cellular survival, track structure theory has been shown to represent an adequate model for cell killing by high-LET radiations of demonstrated predictive value (Katz and Sharma, 1973; Roth and Katz, 1980). Although mechanisms leading to other biological end points may be more complex, experimental data suggest that the general pattern of radiation response is similar to that for cellular inactivation.

\section{Region of Validity of Dose}

To consider the validity of the concept of dose for low-LET radiations of different initial energy spectra, we must discuss the concept of the electron slowing down spectrum (Spencer and Fano, 1954) in relation to electron impact ionization cross sections (Kieffer and Dunn, 1966).

The electron slowing down spectrum is a plot of the sum of all path-length segments in a specified energy interval from all primary and higher generation electrons, as a function of electron energy. When normalized to unit energy, so that radiations of different initial energies can be compared, it is plotted as cm/
$\mathrm{eV}$ (of accumulated segments) per eV (of initial energy), or $\mathrm{cm} / \mathrm{eV}^{2}$. Such spectra are shown in Figure 1, for electrons of initial energy from $1 \mathrm{keV}$ to $1 \mathrm{MeV}$ (Hamm et al. 1978). Similar calculations have been made for photons (Turner et al. 1978).

Plots of electron impact ionization cross sections for $\mathrm{Hg}$ and for molecular $\mathrm{H}$ are shown in Figure 2, as typical for a wide variety of substances. The cross section is small at energies of $10^{4} \mathrm{eV}$ and higher, and increases with a decrease in electron energy to a maximum at about $100 \mathrm{eV}$, and then declines with a further decrease in electron energy to a threshold at the ionization potential. The dominant part of the slowing down spectrum will therefore be between about 10 and $1000 \mathrm{eV}$. When the normalized slowing down spectra of low-LET radiations of different initial energies are congruent in this energy interval, we can expect dose to be a good parameter; that is, the RBE will be equal to 1 . This is the case for electrons of initial energy between $100 \mathrm{keV}$ and $10 \mathrm{MeV}$. At about $10 \mathrm{MeV}$ there is a problem with photonuclear processes. As the energy declines below $100 \mathrm{keV}$ to 100 $\mathrm{eV}$ (Berger 1981), the departure from congruence becomes acute.

To find the number of ionizations produced by an electron of specified initial energy we must sum over

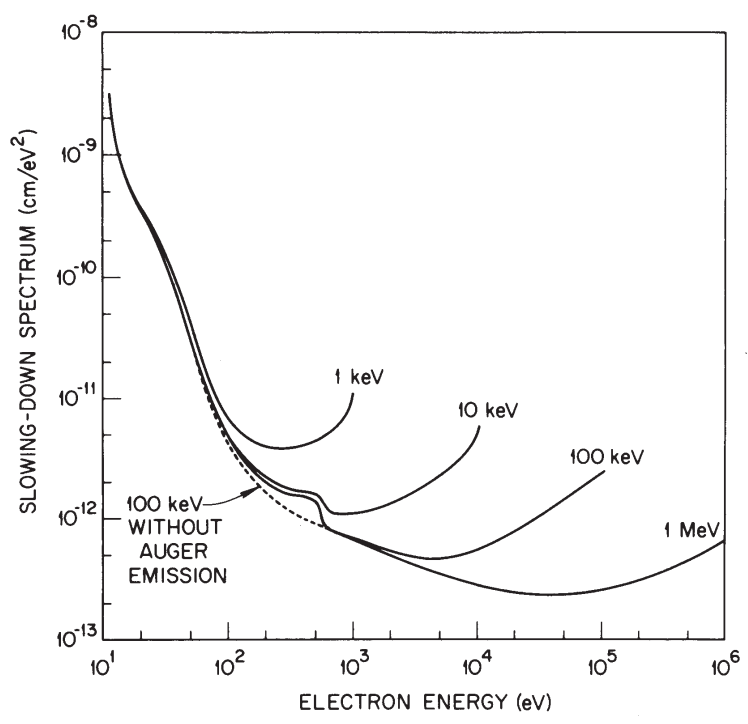

Figure 1. Electron slowing-down spectra in liquid water for electrons of initial energy 1, 10, $100 \mathrm{keV}$, and $1 \mathrm{MeV}$ (Hamm et al. 1978). 

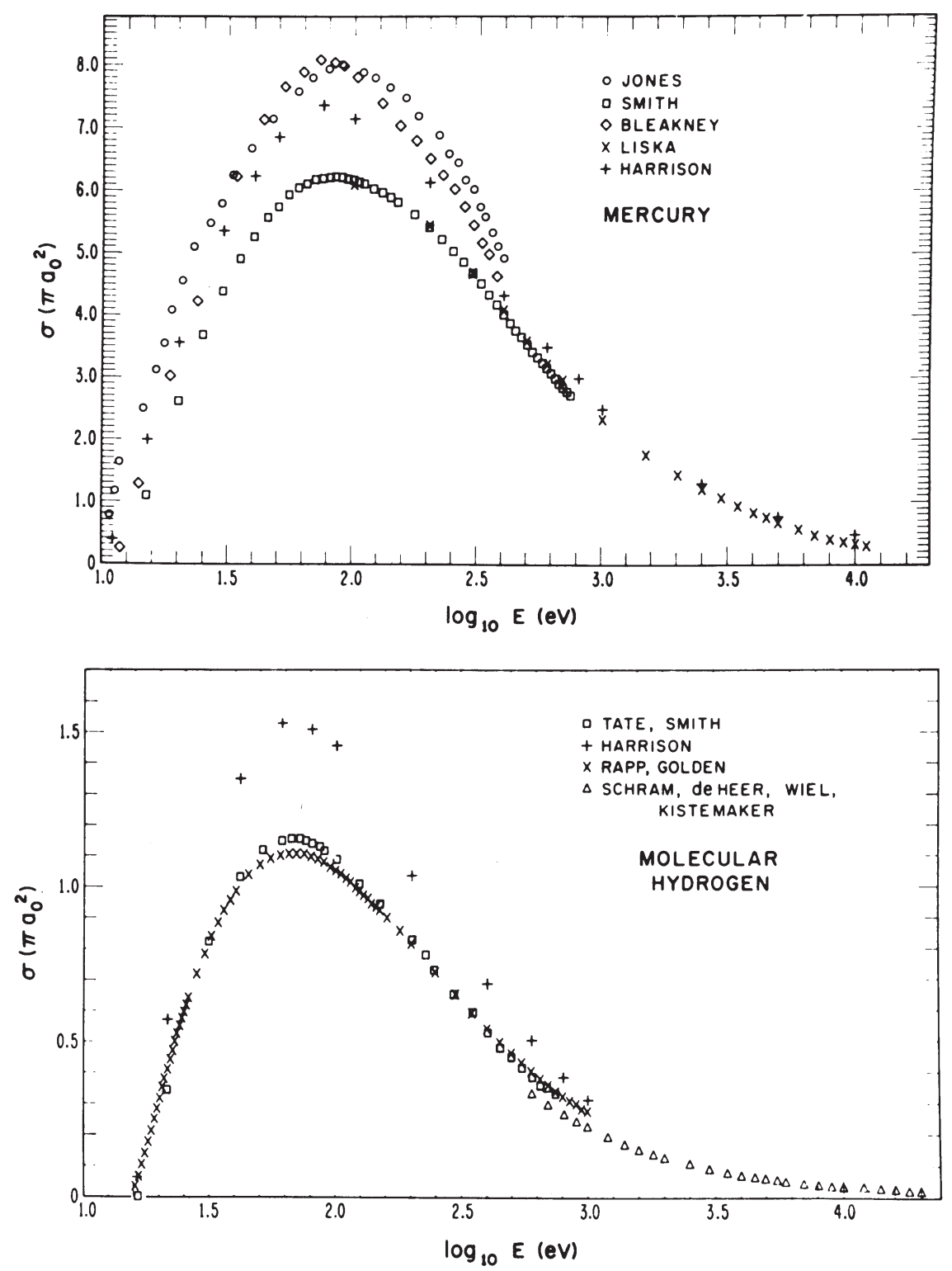

Figure 2. Electron impact ionization cross sections for $\mathrm{Hg}$ and molecular $\mathrm{H}$. See Kieffer and Dunn 1966 for references.

the entire slowing down spectrum. At each electron energy the product of the cross section and the number of atomic targets per unit volume is the number of ionizations per unit path length. This must be multiplied by the path length at this energy. Variations in the dose-level affect the electron fluence, the total path length, but not the shape of the spectrum.
The differences in the shape of the electron slowing down spectra together with the mean spacing of the ionizations relative to the sensitive elements of the detector (Berger 1981) may help to explain why low-LET radiations of low initial energy, $x$ rays from low $Z$ atoms, have RBE's greater than 1 (Goodhead 1980). Since both considerations can be expected to 
play a role, we might expect that the dose of low-LET radiations might be a better index of response for a detector whose sensitive elements are of molecular size than for one whose sensitive elements are much larger. Thus, in contrast to the variation in response of biological systems with energy, no such variation has been found for the alanine dosimeter (Bermann et al. 1971).

\section{High-LET Radiations}

For low doses of high-LET radiations, the effects are produced by isolated individual particle tracks. We make use of the concept of an action cross section, which refers to the probability that a specific end point will be attained as a result of the interaction of the projectile and the target, after a long and generally unknown sequence of internal interactions. After years of study we still do not know precisely what constitutes cellular inactivation or cellular transformation, nor the several pathways through which these end points are achieved. Here we refer to a process called "ion-kill" or "intra-track" effects. Secondary electrons resulting from the passage of the ion through the medium, the $\delta$ rays, are principally responsible for the observed action. The action cross section, $\sigma$, includes the effect produced by all the $\delta$ rays. The product of the action cross section and the number of targets per unit volume, $N$, the quantity $\sigma N$, is the number of targets per unit path length in which the end point is attained. In a nuclear emulsion, this represents the number of grains per unit path length in which a latent image is created. In a biological cell, the action cross section may reflect the probability that some number of internal targets are affected, and so may be altered by changes in the shape of a cell nucleus from spherical to flattened ellipsoid (Lloyd et al. 1979). Where the radiation field at low doses consists of a spectrum of primary and higher generation heavy ions, the ion slowing down spectra, the path lengths, must be multiplied by the number $\sigma N$ per unit path length to find the number of affected cells.

With 1-hit detectors that do not accumulate sub-lethal damage, the transition from low to high dosefrom isolated single tracks to an ensemble of intersecting tracks - is made in the normal way using the cumulative 1 -or-more hit Poisson distribution to correct for track overlap.
Detectors that accumulate sub-lethal damage, e.g. 2-or-more hit, require special consideration at high doses where $\delta$ rays from different ions may intersect in the target. Here we refer to a process called " $\gamma$-kill" or "inter-track" effects. We must be alert to the possibility of characteristic repair or decay times for sublethal damage which might lead to dose-rate effects for $\gamma$-kill.

In the ion-kill mode we refer to normal probability. In the $\gamma$-kill mode we refer to conditional probability, for the effect of $\delta$ rays from a second ion can only be observed if $\delta$ rays from a first ion have penetrated the target. In the ion-kill mode the position of the particle in the detector is random for uniform irradiation, but the sequence of activated targets is not random, for it is constrained to lie along the path of the particle. With $\gamma$-kill, the inactivations are associated with path intersections and are thus truly random. This is illustrated in Figure 3 in which two different emulsions are exposed to an $\alpha$ particle source. One of these, Kodak NTA, ${ }^{1}$ is a 1-hit detector, showing single-particle tracks, while the other, Ilford $\mathrm{K}$ minus $\mathrm{l}^{2}$ is a manyhit detector capable only of showing $\alpha$-particle track intersections.

In considering the effects of high doses of highLET radiations on targets capable of accumulating sub-lethal damage, we make the approximation that one minus the probability of ion-kill is the fraction of the dose delivered in the $r$-kill mode [see equation (3), Table 1]. The initial population of targets for the $\gamma$-kill mode is approximated as the number surviving ion-kill inactivation. This population is reduced as if the $\gamma$-kill dose is made up of $\gamma$ rays. For the calculation of the surviving fraction [see equation (5), Table $1]$, it is assumed that $\gamma$-kill and ion-kill are statistically independent events, i.e., sublethal damage caused by $\gamma$-kill does not affect the ion-kill probability. In this approximation we neglect differences in the slowing down spectra of $\delta$ rays and $\gamma$ rays. In a radiation field consisting of a mixture of high and low-LET radiations, as in the case of neutrons contaminated with $\gamma$ rays, the partial dose of $\gamma$ rays is added to the $\gamma$-kill dose to calculate the survivors of the $\gamma$-kill irradiation [see equation (11), Table 1].

${ }^{1}$ Kodak Nuclear Track Plates, Type NTA, Eastman Kodak Co., Rochester, NY 14650.

2 Ilford Nuclear Research Plate, Type K minus 1, Ilford Ltd., Basildon, Essex, England. 


\section{NTA Exposed $15 \mathrm{~min} D 19$ I:I $5 \mathrm{~min}$}

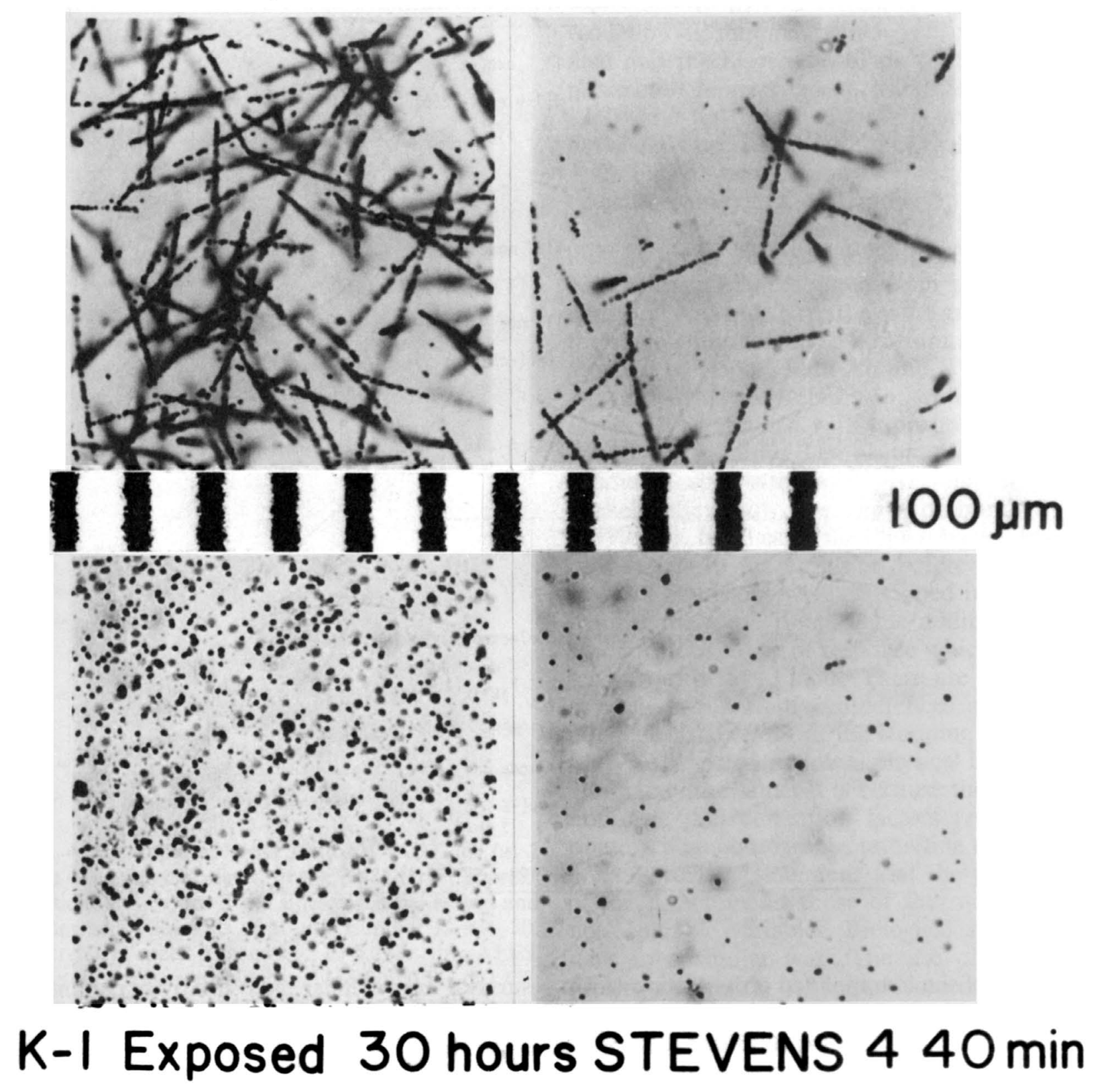

Figure 3. The center (left) and periphery (right) of an $\alpha$-particle source were used to irradiate a sensitive 1-hit emulsion (Kodak NTA, top) and a desensitized many-hit emulsion (K-l, bottom), showing single particle tracks (top) and track intersections (bottom).

These considerations make it clear that only in the $\gamma$-kill mode-hence for many-hit detectors bombarded with fast protons - is dose a good measure of response. Gamma-kill dose is equivalent to $\gamma$-ray dose. In these circumstances the RBE approximates 1 , and the slope of a (log-log) plot of the extrapolated cross section (from the final slope of a survival curve) vs LET approximates 1, as shown in Figure 4. 
Table 1. Collected formulas of the track theory of cellular survival

Track Segment Bombardment

Probability for ion-kill

Effective charge

Gamma-kill dose

Heavy ion dose

Surviving fraction

Survival probability in the gamma-kill mode

Survival probability in the ion-kill mode

Ion-kill cross section

$$
\begin{aligned}
& P=\left[1-\exp \left(-z^{2} / \kappa \beta^{2}\right)\right]^{m} \\
& z=Z\left[1-\exp \left(-125 \beta Z^{-2 / 3}\right)\right] \\
& D_{\gamma}=(1-P) D \\
& D=F L \\
& N / N_{0}=\Pi_{i} \times \Pi_{\gamma} \\
& \Pi_{\gamma}=1-\left[1-\exp \left(-D_{\gamma} / E_{0}\right)\right]^{m} \\
& \Pi_{i}=\exp (-\sigma F) \\
& \sigma=\sigma_{0} P
\end{aligned}
$$

particle fluence $F$, relative speed $\beta$, LET $L$, atomic number $Z$

Cell parameters: $E_{0}, m, \kappa, \sigma_{0}$

Mixed Radiation Field

Total dose

$$
\begin{aligned}
& \mathcal{D}=D+d \\
& D=\sum_{j} \sum_{k} F_{j k} L_{j k} \\
& d
\end{aligned}
$$

Heavy ion dose

Dose of gamma rays, muons, energetic electrons

Gamma-kill dose

$D_{\gamma}=\sum_{j} \sum_{k} F_{j k} L_{j k}\left(1-P_{j k}\right)+d$

Survival probability in the gamma-kill mode,

Equations 11 and 6.

Survival probability in the ion-kill mode

$$
\Pi_{i}=\exp \left[-\sum_{j} \sum_{k} \sigma_{j k} F_{j k}\right]
$$

$j, k$ designations for particles of type $j$ moving at speed $\beta_{k}$.

Except for this limiting circumstance, response is a multiple valued function of dose with high-LET radiations when we consider different bombarding particles. If we are to predict response, we must know the action cross sections and the particle-energy spectra. These spectra are presently only available for neutrons (Caswell and Coyne 1972) and are presently being studied for heavy ion beams (Schimmerling et al. 1983). Where the particle-energy spectra and the low-LET contamination are known, the equations of Table 1 make it possible to calculate cellular survival from cellular radiosensitivity parameters (Katz and
Sharma, 1973; Katz and Sharma 1974; Roth and Katz, 1980).

\section{Correction Factors}

Except for the island of radiation quality, which includes electrons and photons from about $100 \mathrm{keV}$ to $10 \mathrm{MeV}$, and protons from about $10 \mathrm{MeV}$ to $1 \mathrm{GeV}$ (where the RBE is approximately equal to one), response is a multiple valued function of dose with radiation fields of different quality. It has been the general practice to seek a single-parameter reduction 


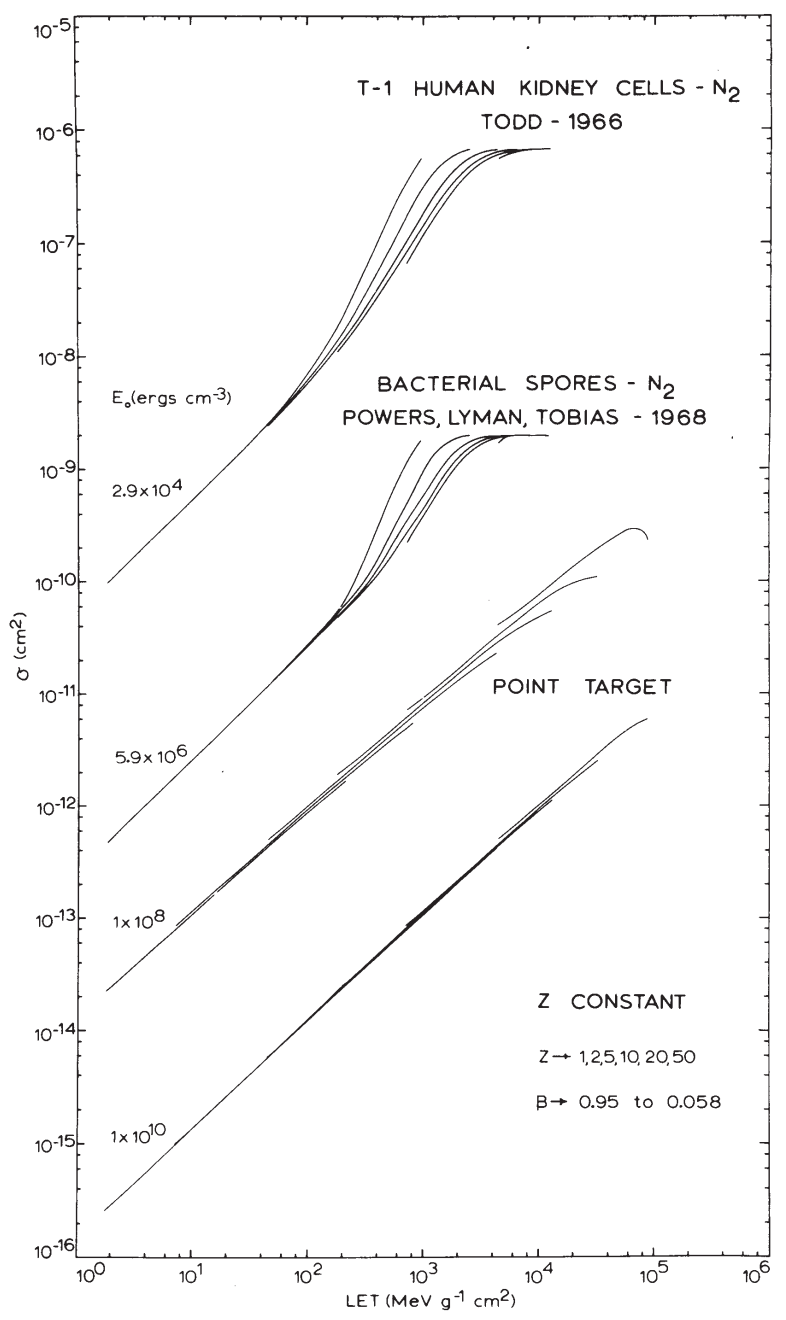

Figure 4. Extrapolated cross section vs LET for (a) 2 1-hit detectors calculated in the point-target approximation, and (b) for 2 cell lines (bacterial spores and kidney cells), for ions of atomic number $Z=1-50$, and relative speed $\beta$ from 0.95 to 0.058 . At low LET the cross sections are single-valued functions of LET. At low-LET, spores and kidney cells are inactivated in the $\gamma$-kill mode so that the curves have slope 1 . At higher LET, ionkill dominates and the curves become multiple valued.

of the radiation field against which to represent a correction factor. Such parameters as the average LET, the average restricted LET, the momentum transfer, $z^{* 2} / \beta^{2}$, the microdosimetric $Y$ and $Z$ all have had only modest success.

The interactions between the detector and the radiation field are so complex that it is impossible to find a single-parameter reduction of the radiation field which will enable us to eliminate this multiplevaluedness. This is because the parameters repre- senting the field and those representing the detector are not separable variables. At the very least a twoparameter reduction is required, which reflects both the field and the specified end point. Such a two-parameter representation using an "effective LET" and an "effective ion-kill dose fraction" has already been achieved for cell killing (Katz et al. 1976).

In Figure 5 we illustrate the inseparability of detector parameters and radiation parameters with a series of photographs of the tracks of Ar ions in Ilford nuclear emulsions. Plates of the $\mathrm{K}$ series are manufactured from a single K.0 emulsion and thus have the same grain size and the same grain density. Maximum sensitivity is attained by doping with $\mathrm{Au}$ and $S$ to about 25 ppm, to obtain K.5 emulsion, while minimum sensitivity is attained with a proprietary desensitizer with $\mathrm{K}$ minus 3 emulsion. All plates were irradiated with a beam of Ar ions from the Berkeley Bevalac ${ }^{3}$ at energies of hundreds of $\mathrm{MeV} /$ amu. In all cases the ions travelled several centimeters in the emulsion before coming to rest. All emulsions were developed in the same bath at the same time. These additives create an enormous range of response which does not scale from plate to plate. There are qualitative as well as quantitative differences in response at equal distances from the stopping end of the track where all of the initial physical interactions are exactly the same. It is because the tracks do not scale that correction factors are inappropriate. These photographs underline the validity of our previous statement that the parameters of the detector and those of the radiation are not separable variables. Biological systems behave in a similar way. The use of a quality factor in radiation assumes incorrectly that the response of biological systems scales according to a single-parameter reduction of the radiation field, the LET. This inherent error cannot be adjusted by changes in the numerical value of the quality factor.

\section{Implications for Radiation Protection}

There is no problem in the use of dose and dose equivalent for those cases where the quality factor is close to 1 , as in the case of photons and electrons of

\footnotetext{
${ }^{3}$ Bevalac accelerator, Lawrence Berkeley Laboratory, University of California, Berkeley, CA 94720.
} 


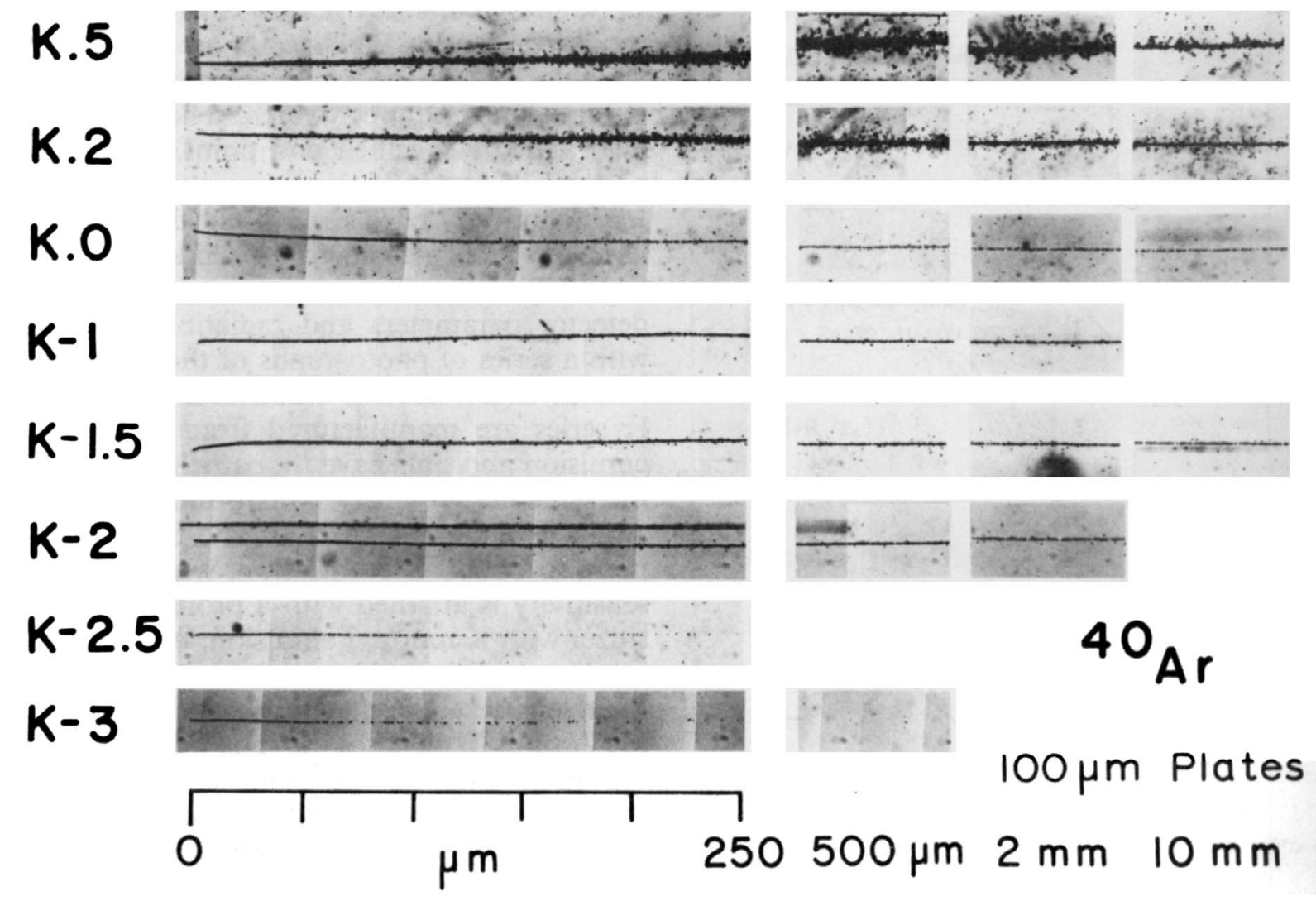

Figure 5. Tracks of Ar ions in Ilford K emulsions. The ions enter the emulsions at right and stop at left. Shown are short segments at 10, $2 \mathrm{~mm}, 500$ and $250 \mu \mathrm{m}$. The top 6 tracks, from K.5 to K-2, are characteristic of 1-hit detectors with different values of $E_{0}$. The 2 bottom tracks are characteristic of many hit detectors.

initial energy between $100 \mathrm{keV}$ and $10 \mathrm{MeV}$, or in the case of fast protons. In these instances dose is a good index of response.

For other radiations, like neutrons or $\alpha$ particles, or HZE particles, or these mixed with $\gamma$-ray contamination, another procedure is suggested. We suggest that a specific end point be selected and that the quality factor be defined as the RBE for that end point under defined experimental conditions. Because of our current limitation to cellular survival, we propose to define this quality factor as the RBE for in vitro cellular inactivation of a specific cell line representative of human tissue, e.g. T-1 kidney cells under aerobic conditions.

The present state of radiobiological knowledge limits our treatment to cellular survival. Calculations of cellular survival have been shown to agree with RBE measurements for tissues with neutrons of different energies (Katz and Sharma 1975). Only limited data are available from which to extract radiosensitivity parameters for mutation induction and cell transformation. Where possible this has been done (Hofmann and Katz 1983). If these were available, one could base estimates of radiation quality on a calculation of mutations or transformations per surviving cell. Limited experimental information on mutation induction and transformation suggests that the RBE vs LET curves for these end points are qualitatively and quantitatively similar to that for cell killing, particularly for a given cell line (Goodhead 1984; Hofmann and Katz 1983). In the absence of such knowledge our discussion will focus on cellular survival, 
for which the formula of Table 1, and known cellular radiosensitivity parameters have formed a predictive combination (Roth et al. 1976).

Calculations of cellular survival for complex radiation fields tend to be quite involved. However, it has been shown that a two-parameter reduction of the interaction of the field with a specific cellular system (represented by radiosensitivity parameters) can be made. We can calculate, or measure, the quantities $P$ (the ion-kill probability) and $L$ (the effective LET). From these quantities and the cellular radiosensitivity parameters, we can calculate the fraction of surviving cells as a function of dose. With dosimeter badges sensitive to different components of a radiation field, we conceive that values of $P$ and $L$ can be assigned to each separate component of the badge. Theory allows us to combine the effects of these fractions (Katz et al. 1976). Such calculations are readily programmed for hand-held calculators. The availability of these devices enables us to consider more complicated computational algorithms than one restricted to multiplication and addition.

\section{References}

Berger M. J., 1981, “On the spatial correlation of ionization events in water," in: Proc. 7th Symp. on Microdosimetry, p. 521 (Chur, Switzerland: Harwood Academic).

Bermann F., de Choudens H., and Descours S., 1971, “Application à la dosimetrie de la mesure par RBE des radicaux libres créés dans les acides aminés," in: IAEA Symp. Advances in Physical and Radiation Detectors, p. 311 (Vienna: IAEA).

Caswell R. S. and Coyne J. J., 1972, “Interaction of neutrons and secondary charged particles with tissue: secondary particle spectra," Radial. Res. 23,1149.

Goodhead D. T., 1980, "Models of radiation inactivation and mutagenesis," in: Radiation Biology and Cancer Research, p. 231 (New York: Raven Press).
Goodhead D. T., 1984, “Deductions from cellular studies of inactivation, mutagenesis, and transformation," in: $R a-$ diation Carcinogenesis: Epidemiology and Biological Significance, p. 369 (New York: Raven Press).

Hamm R. N., Wright H. A., Katz R., Turner J. E., and Ritchie R. H., 1978, "Calculated yields and slowing-down spectra for electrons in liquid water: implications for electron and photon RBE," Phys. Med. Biol. 23, 1149.

Hofmann W. and Katz R., 1983, "Particle fluence and radiation carcinogenesis," in: Proc. 7th Int. Congr. Radiation Research, C8-07 (Amsterdam: Martinus Nijhoff).

Katz R. and Sharma S. C., 1973, "Response of cells to fast neutrons, stopped pions, and heavy ion beams," $\mathrm{Nucl}$. Instrum. Meth. 110, 93.

Katz R. and Sharma S. C., 1974, “OER for mixed neutrons and gamma-rays," Br. J. Radio. 47, 823.

Katz R. and Sharma S. C., 1976, "RBE-dose relations for neutrons and pions," Phys. Med. Biol. 20, 410.

Katz R., Fullerton Jr. B. G., Roth R. A., and Sharma S. C., 1976, "Simplified RBE-dose calculations for mixed radiation fields," Health Phys. 30, 148.

Kieffer L. J. and Dunn G. H., 1966, “Electron impact ionization cross section data for atoms, atomic ions, and diatomic molecules: I. Experimental data," Rev. Mod. Phys. 38, 1.

Lloyd E. L., Gemmel M. A., Henning C. B., Gemmel D. S., and Zabransky B. J., 1979, "Cell survival following multiple track alpha particle irradiation," Int. J. Radiat. Biol. $35,22$.

Roth R. A., Sharma S. C., and Katz R., 1976, "Systematic evaluation of cellular radiosensitivity parameters," Phys. Med. Biol. 21, 491.

Roth R. A. and Katz R., 1980, “Heavy ion beam model for radiobiology," Radiat. Res. 83, 499.

Schimmerling W., Subramanian T. S., McDonald W. J., Kaplan S. N., Sadoff A., and Gabor G., 1983, "Beam analysis spectrometer for relativistic heavy ions," Nucl. Instrum. Meth. 205, 531.

Spencer L. V. and Fano U., 1954, "Energy spectrum resulting from electron slowing down," Phys. Rev. 93, 1172.

Turner J. E., Hamm R. N., Wright H. A., Ritchie R. H., and Katz R., 1978, “Electron slowing-down spectra for liquid water irradiated by $\mathrm{x}$ and gamma rays-implications for photon RBE," in: Proc. 6th Symp. on Microdosimetry, p. 375 (London: Harwood Academic Publishers). 